\title{
SCIENTIFIC REPORTS

\section{OPEN Epidural bolus versus continuous epidural infusion analgesia on optic nerve sheath diameter in paediatric patients: A prospective, double- blind, randomised trial}

Bora Lee $\mathbb{D}^{1}$, Jae Hoon Lee $\mathbb{D}^{1}$, Min-Soo Kim ${ }^{1}$, Seon Ju Kim ${ }^{1}$, Jeehyun Song ${ }^{1}$, Do-Hyeong Kim ${ }^{2}$ \&Yong Seon Choi $\mathbb{1}^{1 *}$

The use of programmed intermittent epidural bolus for postoperative analgesia may have greater analgesic efficacy than continuous epidural infusion. However, the rapid delivery speed used with an epidural bolus is more likely to increase intracranial pressure. We compared the effects of lumbar epidural bolus versus continuous infusion epidural analgesia on intracranial pressure in children using optic nerve sheath diameter as a marker. We randomly assigned 40 paediatric patients to bolus or infusion groups. Epidural analgesia ( $0.15 \%$ ropivacaine $\left.0.3 \mathrm{ml} \cdot \mathrm{kg}^{-1}\right)$ was administered via bolus or infusion. Ultrasonography was used to measure the optic nerve sheath diameter before (TO), at $3 \mathrm{~min}$ (T1), $10 \mathrm{~min}$ (T2), and $70 \mathrm{~min}$ (T3) after starting the pump. There were statistically significant betweengroup differences in optic nerve sheath diameter over time $\left(P_{\text {Group } x T i m e}=0.045\right)$. From T0-T3, the area under the curve values were similar between the two groups. Although there were differences in the patterns of optic nerve sheath diameter change according to the delivery mode, the use of lumbar epidural bolus did not increase the risk of intracranial pressure increase over that of continuous infusion. Further research is needed to investigate intracranial pressure changes after continuous application of each delivery mode.

Inadequate use of analgesia after an operation causes unacceptable pain, generates long-lasting pain memories, and increases the risk of behavioural disorders in paediatric patients ${ }^{1,2}$. When possible, regional analgesia should be performed to optimise postoperative pain management ${ }^{3}$. Continuous epidural catheterisation under general anaesthesia has been used to provide sufficient postoperative analgesia in the days following surgery $y^{3,4}$, and epidural analgesia is effective for postoperative pain control in paediatric patients ${ }^{5-8}$.

Compared with continuous epidural infusion, programmed intermittent epidural bolus can have greater analgesic efficacy and fewer side effects in surgical patients ${ }^{9-11}$. However, an epidural bolus may generate relatively higher injection pressure because the delivery speed of a solution through the epidural catheter is directly related to peak pressures ${ }^{12}$. Thus, compared with continuous epidural infusion, pressure changes after programmed intermittent epidural bolus use are more likely to increase epidural pressure and, subsequently, intracranial pressure. Use of caudal block causes an increase in intracranial pressure in paediatric patients ${ }^{13}$, but the effect of epidural analgesia on intracranial pressure in paediatric patients has not been investigated. Studies of non-invasive methods used for intracranial pressure measurement have demonstrated that optic nerve sheath diameter is correlated with intracranial pressure; optic nerve sheath diameter measurement has high diagnostic accuracy for detection of increased intracranial pressure in children ${ }^{14-16}$. This prospective, randomised, double-blinded study aimed to

\footnotetext{
${ }^{1}$ Department of Anaesthesiology and Pain Medicine, Severance Hospital and Anaesthesia and Pain Research Institute, Yonsei University College of Medicine, 50-1 Yonsei-ro, Seodaemun-Gu, Seoul, 03722, Republic of Korea. ${ }^{2}$ Department of Anaesthesiology and Pain Medicine, Anaesthesia and Pain Research Institute, Gangnam Severance Hospital, Yonsei University College of Medicine, 211 Eonju-ro, Gangnam-gu, Seoul, 06273, Republic of Korea. *email: YSCHOI@yuhs.ac
} 


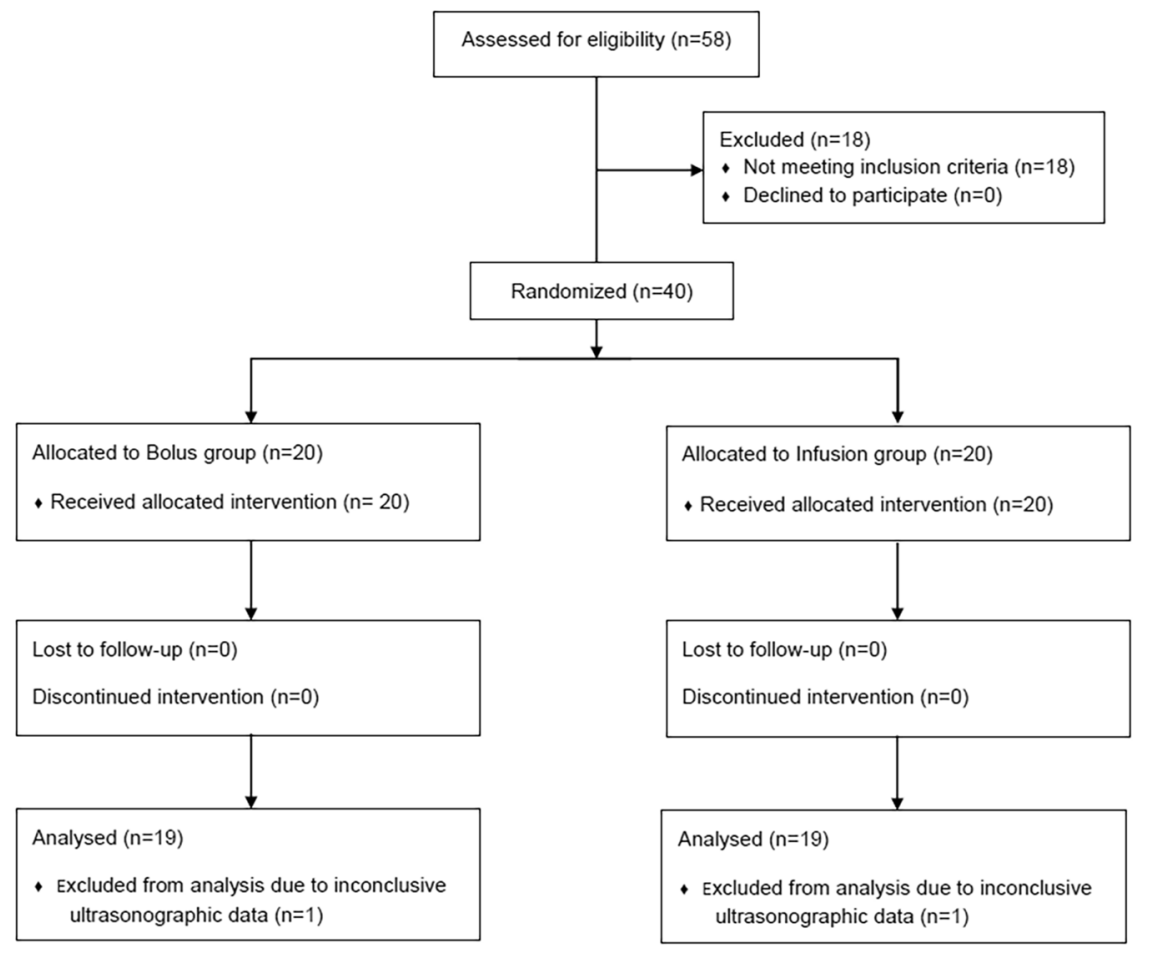

Figure 1. Flow diagram of the study.

\begin{tabular}{|l|l|l|c|}
\hline & $\begin{array}{l}\text { Bolus group } \\
(\mathbf{n = 1 9 )}\end{array}$ & $\begin{array}{l}\text { Infusion group } \\
(\mathbf{n = 1 9 )}\end{array}$ & P value \\
\hline Age (years) & $9.42 \pm 2.95$ & $9.24 \pm 2.62$ & 0.840 \\
\hline Sex (male) & $12(63)$ & $13(68)$ & $>0.999$ \\
\hline Height $(\mathrm{cm})$ & $128.4 \pm 16.7$ & $123.0 \pm 17.7$ & 0.348 \\
\hline Weight $(\mathrm{kg})$ & $29.5 \pm 9.2$ & $26.5 \pm 8.5$ & 0.302 \\
\hline Body mass index $\left(\mathrm{kg} \cdot \mathrm{m}^{-2}\right)$ & $17.4 \pm 3.0$ & $17.5 \pm 4.1$ & 0.898 \\
\hline Anaesthesia time $(\mathrm{min})$ & $306 \pm 119$ & $346 \pm 105$ & 0.278 \\
\hline Operation time $(\mathrm{min})$ & $240 \pm 108$ & $290 \pm 98$ & 0.144 \\
\hline Recovery time $(\mathrm{min})$ & $50.0(42.5-59.0)$ & $42.0(40.0-52.5)$ & 0.395 \\
\hline
\end{tabular}

Table 1. Patient characteristics and operative data in the lumbar epidural bolus and infusion group. Values are presented as mean $\pm \mathrm{SD}$, median (IQR), or number (proportion).

investigate the effects of lumbar epidural bolus and infusion on intracranial pressure using ultrasonographic optic nerve sheath diameter measurements in children.

\section{Results}

Forty of the 58 patients assessed for eligibility were enrolled in the study (August 2017 to May 2018) and were randomly assigned to either the epidural bolus or infusion group. Thirty-eight of the 40 patients completed the study (Fig. 1). Two subjects were excluded from the final analysis because optic nerve sheath borders were not apparent at the follow-up ultrasonographic measurement. The mean value of T1-T0 was $10 \mathrm{~min}$. Patient characteristics and intraoperative variables were similar between the two groups (Table 1).

Table 2 presents the results of analyses of hemodynamic data and data for variables potentially affecting intracranial pressure (e.g., end-tidal carbon dioxide concentration, end-tidal sevoflurane concentration, and peak inspiratory pressure). There were no statistically significant between-group differences among these variables at any time point.

Table 3 presents the mean values for optic nerve sheath diameter from the linear mixed model and the corresponding $\mathrm{P}$ values. The analysis revealed statistically significant between-group differences for changes in optic nerve sheath diameter $\left(\mathrm{P}_{\text {Group } \times \text { Time }}=0.045\right)$ (Fig. 2). The post-hoc analysis revealed that optic nerve sheath diameter at T2 was significantly increased from baseline in the bolus group and that optic nerve sheath diameter at T3 was significantly increased from baseline in the infusion group. Although the patterns of increase in optic nerve sheath diameter differed between the two groups, the maximal increase in optic nerve sheath diameter from 


\begin{tabular}{|c|c|c|c|}
\hline & $\begin{array}{l}\text { Bolus group } \\
(\mathrm{n}=19)\end{array}$ & $\begin{array}{l}\text { Infusion group } \\
(\mathrm{n}=19)\end{array}$ & Pvalue \\
\hline \multicolumn{4}{|c|}{ Heart rate (beats.min ${ }^{-1}$ ) } \\
\hline T0 & $110 \pm 18$ & $109 \pm 18$ & 0.905 \\
\hline $\mathrm{T} 1$ & $103 \pm 16$ & $97 \pm 17$ & 0.269 \\
\hline $\mathrm{T} 2$ & $95 \pm 18$ & $92 \pm 18$ & 0.634 \\
\hline T3 & $97 \pm 19$ & $100 \pm 17$ & 0.618 \\
\hline \multicolumn{4}{|c|}{ Mean arterial pressure $(\mathrm{mmHg})$} \\
\hline T0 & $75.2 \pm 9.9$ & $75.0 \pm 11.9$ & 0.941 \\
\hline T1 & $73.3 \pm 12.5$ & $72.7 \pm 11.0$ & 0.880 \\
\hline $\mathrm{T} 2$ & $69.0(66-78)$ & $77.0(69-80)$ & 0.267 \\
\hline T3 & $75.0 \pm 7.8$ & $75.8 \pm 8.7$ & 0.755 \\
\hline \multicolumn{4}{|c|}{ Peak inspiratory pressure $\left(\mathrm{cmH}_{2} \mathrm{O}\right)$} \\
\hline T0 & $13.1 \pm 1.7$ & $12.0 \pm 1.9$ & 0.080 \\
\hline $\mathrm{T} 1$ & $12.7 \pm 1.7$ & $11.7 \pm 2.2$ & 0.147 \\
\hline $\mathrm{T} 2$ & $12.8 \pm 1.7$ & $11.8 \pm 2.1$ & 0.117 \\
\hline T3 & $13.5 \pm 2.2$ & $12.8 \pm 2.6$ & 0.346 \\
\hline \multicolumn{4}{|c|}{ End-tidal carbon dioxide $(\mathrm{mmHg})$} \\
\hline T0 & $34.4 \pm 1.2$ & $33.8 \pm 1.6$ & 0.220 \\
\hline $\mathrm{T} 1$ & $34.3 \pm 1.9$ & $33.8 \pm 1.3$ & 0.437 \\
\hline $\mathrm{T} 2$ & $33.7 \pm 1.8$ & $34.2 \pm 1.8$ & 0.482 \\
\hline $\mathrm{T} 3$ & $34.0(33.0-35.5)$ & $34.0(33.0-35.5)$ & 0.688 \\
\hline \multicolumn{4}{|c|}{ End-tidal sevoflurane concentration (\%) } \\
\hline T0 & $1.57 \pm 0.45$ & $1.41 \pm 0.36$ & 0.226 \\
\hline $\mathrm{T} 1$ & $1.40(1.20-1.65)$ & $1.20(1.10-1.65)$ & 0.171 \\
\hline $\mathrm{T} 2$ & $1.6(1.25-1.90)$ & $1.60(1.30-1.70)$ & 0.758 \\
\hline T3 & $2.20 \pm 0.28$ & $2.37 \pm 0.35$ & 0.100 \\
\hline
\end{tabular}

Table 2. Hemodynamic data and parameters associated with intracranial pressure at each time point in the lumbar epidural bolus and infusion groups. Values are presented as mean $\pm \mathrm{SD}$ or median (IQR).

\begin{tabular}{|l|l|l|l|}
\hline \multicolumn{2}{|l|}{$\begin{array}{l}\text { Bolus group } \\
(\mathbf{n}=\mathbf{1 9})\end{array}$} & $\begin{array}{l}\text { Infusion group } \\
(\mathbf{n}=\mathbf{1 9})\end{array}$ & P value \\
\hline \multicolumn{3}{|l|}{ Optic nerve sheath diameter $(\mathbf{m m})$} \\
\hline T0 & $5.33 \pm 0.14$ & $5.25 \pm 0.14$ & 0.674 \\
\hline T1 & $5.56 \pm 0.13$ & $5.31 \pm 0.14$ & 0.200 \\
\hline T2 & $5.60 \pm 0.13$ & $5.41 \pm 0.14$ & 0.330 \\
\hline T3 & $5.57 \pm 0.14$ & $5.66 \pm 0.15$ & 0.633 \\
\hline $\begin{array}{l}\text { Changes in optic nerve } \\
\text { sheath diameter }(\mathrm{mm})\end{array}$ & & Adjusted P value \\
\hline T1-T0 & $0.23 \pm 0.09$ & $0.07 \pm 0.09$ & $>0.999$ \\
\hline T2-T0 & $0.27 \pm 0.09 *$ & $0.16 \pm 0.09$ & $>0.999$ \\
\hline T3-T0 & $0.24 \pm 0.1$ & $0.41 \pm 0.11 *$ & $>0.999$ \\
\hline T2-T1 & $0.03 \pm 0.09$ & $0.09 \pm 0.09$ & $>0.999$ \\
\hline T3-T1 & $0 \pm 0.1$ & $0.34 \pm 0.12 *$ & 0.0498 \\
\hline T3-T2 & $-0.03 \pm 0.1$ & $0.25 \pm 0.11$ & $>0.999$ \\
\hline
\end{tabular}

Table 3. Changes in optic nerve sheath diameter between time points in the lumbar epidural bolus and infusion group. Values are presented as estimated mean \pm standard error from the linear mixed model. Adjusted $\mathrm{P}$ value indicates the Bonferroni-corrected $\mathrm{P}$ value. *Adjusted $P<0.05$ in each group.

baseline was not statistically significant between the groups (bolus group: 0.27 ; infusion group: $0.41 ; \mathrm{P}>0.999$ ). The areas under the curve for T0-T3 were similar for the two groups.

\section{Discussion}

This study is the first to investigate the effects of lumbar epidural analgesia on intracranial pressure comparing the use of bolus and infusion protocols in paediatric patients. Our results revealed a faster increase of optic nerve sheath diameter in the bolus group compared with the infusion group. Although there were differences in the patterns of optic nerve sheath diameter change according to the method of injection, the total optic nerve sheath diameter changes were not significantly different between the two groups. 


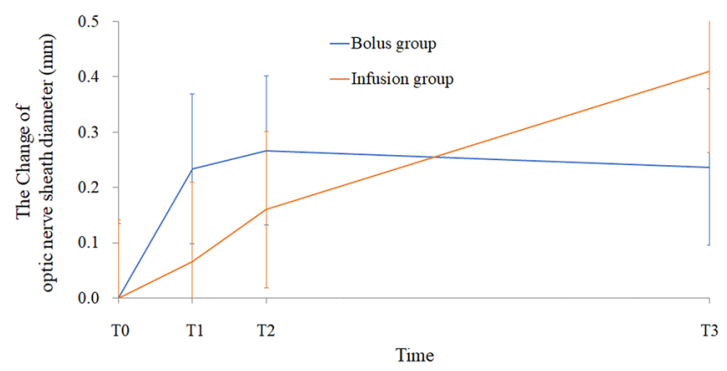

Figure 2. Changes in optic nerve sheath diameter. Values are expressed as estimated mean \pm standard error values from linear mixed model. *The linear mixed model revealed that there were statistically significant between-group differences in changes in ONSD according to time (model corrected for age, gender, height, weight, end-tidal sevoflurane and carbon dioxide levels, and peak inspiratory pressure; $\mathrm{P}_{\text {Group } \times \text { Time }}=0.045$ ). T0, before starting the pump; T1, 3 min after starting the pump; T2, $10 \mathrm{~min}$ after starting the pump; T3, $70 \mathrm{~min}$ after starting the pump.

The prevalence of moderate-to-severe pain in paediatric patients remains high ${ }^{17}$. Use of regional analgesia may play a major role in pain score reduction ${ }^{3}$. Epidural patient-controlled analgesia can be used for paediatric patients in major thoracic, abdominal, or orthopaedic surgeries ${ }^{3,4}$. In particular, effective epidural analgesia prevents muscle spasms that are primarily a spinal reflex caused by pain in children with cerebral palsy who undergo extensive lower limb orthopaedic surgery ${ }^{8}$. Epidural injection provides analgesia via the spread of local anaesthetic through the noncontinuous epidural space. The distribution of local anaesthetics into the epidural space is not uniform and is directed into multiple parallel passages ${ }^{18}$. A more uniform and extensive spread occurs when large volumes and high injectable pressures are administered ${ }^{18,19}$. With the development of patient-controlled analgesia units, the injection mode can be controlled using programmed intermittent epidural bolus or continuous epidural infusion ${ }^{20}$. After posterior spinal fusion for adolescent idiopathic scoliosis, cumulative opioid consumption is lower when the intermittent bolus function is used than with the continuous infusion mode; rates of postoperative nausea, vomiting and pruritus are much greater when the continuous infusion mode is used ${ }^{11}$. Use of programmed intermittent epidural bolus reduces anaesthetic consumption during labour analgesia and decreases labour pain and motor block $\mathrm{k}^{9,10,21,22}$. However, bolus injection into a confined space may increase epidural pressure and intracranial pressure, especially in children.

The variation in intracranial pressure correlates with the optic nerve sheath diameter because the optic nerve is surrounded by distensible subarachnoid space ${ }^{23}$. Hansen et al. found that changes in optic nerve sheath diameter are closely associated with intracranial pressure variations within a limited intracranial pressure interval $(20-50 \mathrm{mmHg})$, and ongoing enlargement of optic nerve sheath diameter on serial ultrasonography has clinical relevance ${ }^{24}$. Several studies of the relationship between optic nerve sheath diameter and intracranial pressure have shown that optic nerve sheath diameter is a useful method for detecting elevated intracranial pressure in children and adults ${ }^{15,25-31}$.

The optic nerve sheath diameters were compared before starting the pump, and $3 \mathrm{~min}, 10 \mathrm{~min}$, and $70 \mathrm{~min}$ after starting the pump between the single bolus and continuous infusion protocols outlined in this study. The second optic nerve sheath diameters were measured at $3 \mathrm{~min}$ after the pump was started because it took up to $3 \mathrm{~min}$ for the bolus injection to reach completion in the bolus group. Previous studies have found that optic nerve sheath diameter increases to a peak value approximately $10 \mathrm{~min}$ after an increase in epidural pressure and intracranial pressure ${ }^{13}$. Based on this, optic nerve sheath diameter was measured at the times of 10 min after starting the pump in the bolus group and $10 \mathrm{~min}$ after completion of continuous infusion in the infusion group. In the bolus group, as we expected, the maximum value was observed $10 \mathrm{~min}$ after the epidural bolus was administered, rather than immediately afterward. We also expected that the lumbar epidural bolus would result in a greater increase in optic nerve sheath diameter than would continuous infusion because the delivery speed was much faster in the bolus group ${ }^{12}$. However, although the optic nerve sheath diameter rapidly increased in the bolus group, epidural bolus did not cause a greater increase in optic nerve sheath diameter than did infusion. There were continuous increases in the optic nerve sheath diameter in the infusion group for $70 \mathrm{~min}$ that were similar in degree to those observed in the bolus group. The total burdens of optic nerve sheath diameter (areas under the curve) were not significantly different between the two groups. This result suggests that the risks of intracranial pressure increases were similar between groups and that the bolus group was not associated with a greater risk of intracranial pressure increase than was the infusion group. The rates of nausea and vomiting are higher during the postoperative period among patients exposed to continuous epidural infusion than among those receiving programmed intermittent epidural bolus analgesia in the postoperative period ${ }^{10,11}$. Unless there is a significant difference in the risk of elevating intracranial pressure between the two modes, the use of programmed intermittent epidural bolus may be preferred because it results in fewer side effects.

Padayachy et al. found that an optic nerve sheath diameter $>5.75 \mathrm{~mm}$ has the best diagnostic accuracy for detecting an intracranial pressure $>20 \mathrm{mmHg}$ in children $>1$ year of age ${ }^{31}$. Compared with this threshold, the baseline values of optic nerve sheath diameter in some subjects of our study were quite elevated. However, the median age of the previous study population was 36 months and the mean age of subjects in our study was 9 years. This is important to note because between the ages of four and five, the optic nerve sheath grows to its adult size ${ }^{27}$. In a paediatric population with a similar mean age as in our study, the optic nerve sheath diameter measured 
using ultrasonography was $5.75 \pm 0.52$ (mean $\pm \mathrm{SD})^{32}$, which was comparable to the baseline optic nerve sheath diameter observed in our study population.

The limitations of this study are as follows. First, we observed the optic nerve sheath diameter for up to $70 \mathrm{~min}$ after applying the two epidural analgesia modes for $1 \mathrm{~h}$. Thus, it is difficult to predict whether optic nerve sheath diameter continues to rise after the 70-min period observed in this study. Further research is needed to investigate how optic nerve sheath diameter changes after continuous application of both delivery modes. Second, the children who were included as subjects were undergoing osteotomy of the legs caused by cerebral palsy. However, we excluded patients with brain lesions or a history of increased intracranial pressure. Normal intelligence occurs in $>60 \%$ of children with hemiplegia ${ }^{8}$, and the baseline optic nerve sheath diameter observed in our study was comparable to the distribution of optic nerve sheath diameter in unaffected children ${ }^{32}$. Thus, the results of this study are expected to be similar to those of the general paediatric population. Third, although the volume of saline administered was small $(<2 \mathrm{ml})$, saline injected into the epidural space during loss of resistance confirmation could affect optic nerve sheath diameter.

In conclusion, although there were differences in the patterns of optic nerve sheath diameter change according to the lumbar epidural injection method, the bolus group was not associated with a greater risk of intracranial pressure increase than was the infusion group. Further research is needed to investigate intracranial pressure changes after continuous application of each delivery mode.

\section{Methods}

Patients. The Severance Hospital institutional review board (protocol number: 4-2017-0341) approved the study protocol (ClinicalTrial.gov, NCT03200951, 27/06/2017). This study was performed in accordance with relevant guidelines and regulations. Written informed consent was obtained from the children who could express consent and from the parents of all children. Forty patients (aged 4-14 years) among children undergoing lower extremity orthopaedic surgery at Severance Hospital who had body weights $\leq 40 \mathrm{~kg}$ and who had treatment plans for epidural analgesia were enrolled. The body weight limit was set to reduce variations in patient physique. Patients were excluded from the study if they had one or more symptoms or clinical signs of spinal anomalies or infection, coagulopathy, ophthalmic disease, increased intracranial pressure (baseline optic nerve sheath diameter $>6.27 \mathrm{~mm})^{32}$, history of increased intracranial pressure (e.g., ventriculoperitoneal shunt, hydrocephalus) or an expected duration of anaesthesia $<70 \mathrm{~min}$.

Anaesthesia. Anaesthesia was induced with propofol (Fresofol 1\% MCT, $2 \mathrm{mg} \cdot \mathrm{kg}^{-1}$; Fresenius Kabi Austria $\mathrm{GmbH}$, Graz, Austria), rocuronium (Rocumeron, $0.8 \mathrm{mg} \cdot \mathrm{kg}^{-1}$; Ilsung Pharmaceuticals Co., Ltd., Seoul, Korea), and remifentanil (Ultian, 0.5-1 $\mu \mathrm{g} \cdot \mathrm{kg}^{-1}$; Hanlim Pharm. Co., Ltd., Seoul, Korea). After intubation, anaesthesia was maintained using sevoflurane in oxygen/air (40\%) and remifentanil $\left(0.05-0.15 \mu \mathrm{g} \cdot \mathrm{kg}^{-1} \cdot \mathrm{min}^{-1}\right)$ to keep the bispectral index level within 40-60. All patients were mechanically ventilated in order to maintain $35-40 \mathrm{mmHg}$ of end-tidal carbon dioxide $\left(\mathrm{EtCO}_{2}\right)$ at a tidal volume of $8 \mathrm{ml} \cdot \mathrm{kg}^{-1}$, and a positive end-expiratory pressure of $3 \mathrm{~cm}$ $\mathrm{H}_{2} \mathrm{O}$ was applied.

A radial artery catheter and a peripheral intravenous line were placed after induction. Pulse oximetry, electrocardiography, gas analysis, invasive arterial blood pressure, oropharyngeal temperature, bispectral index, and $\mathrm{EtCO}_{2}$ concentration were used as standard monitors.

Assignments and interventions. The surgeons, patients, and anaesthesiologist responsible for optic nerve sheath diameter measurement were all blinded to the group assignments during the entire study period. A computer-generated randomisation table (http://www.random.org) was used to assign 40 patients to the epidural bolus or infusion groups. The principal investigator (Y.S.C.) performed the randomisation and ensured the correct assignment for each patient. In the bolus group, the pump (Accumate $1200 \mathrm{v} 2.31$, Woo Young Medical Co., Ltd., Seoul, Korea) was programmed to deliver $0.3 \mathrm{ml} \cdot \mathrm{kg}^{-1}$ of $0.15 \%$ ropivacaine at a rate of $250 \mathrm{ml} \cdot \mathrm{hr}^{-1}$. In the infusion group, the pump was programmed to infuse $0.15 \%$ ropivacaine at a rate of $0.3 \mathrm{ml} \cdot \mathrm{kg}^{-1} \cdot \mathrm{hr}^{-1}$. In both groups, the pumps were stopped $1 \mathrm{~h}$ after epidural analgesia. Ultrasonography (SonoSite X-PORTE, FujiFilm SonoSite Korea Ltd., Seoul, Korea) was used to visualise the lumbar vertebrae and epidural space after induction of anaesthesia. The skin-to-epidural space depth was measured with each patient in a left lateral decubitus position. An 18-gauge Tuohy needle was inserted at the L2-3 interspace level, and the epidural space was identified by loss of resistance to saline. A 20 -gauge epidural catheter was inserted $3 \mathrm{~cm}$ upward into the epidural space. The possibility of intravascular placement was excluded using an aspiration test. The principle investigator set up the epidural pumps according to each patient's assigned group. The pump was concealed in an opaque bag until the optic nerve sheath diameter measurements were completed.

Measurement. One investigator (B.L) with experience in over 50 cases of ultrasonographic optic nerve sheath diameter measurement used transorbital sonography to measure optic nerve sheath diameter; this investigator was blinded to group assignment. To reduce the risk of ultrasonic energy-induced eye injury, the ultrasonography was performed using a linear 6- to 13-MHz probe (FujiFilm SonoSite Korea Ltd., Seoul, Korea) with reduced power output (mechanical index $=0.2$; thermal index $=0$ ). After application of a thick sterile coupling gel layer to the closed upper eyelid, the probe was placed without exerting pressure. Axial images of the orbit were acquired in the plane of the optic nerve, and optic nerve sheath diameter was measured $3 \mathrm{~mm}$ posterior to the globe as described previously (Fig. 3) ${ }^{15}$. A $3.0-3.5 \mathrm{~cm}$ required depth was usually used. The optic nerve sheath diameters were measured at four time points: before starting the pump (T0) and $3 \mathrm{~min}$ (T1), $10 \mathrm{~min}$ (T2), and $70 \mathrm{~min}$ (T3) after starting the pump. Two measurements of each optic nerve sheath were acquired for each eye at each time point and these were averaged to obtain the optic nerve sheath diameter for that time point. Heart rate, 


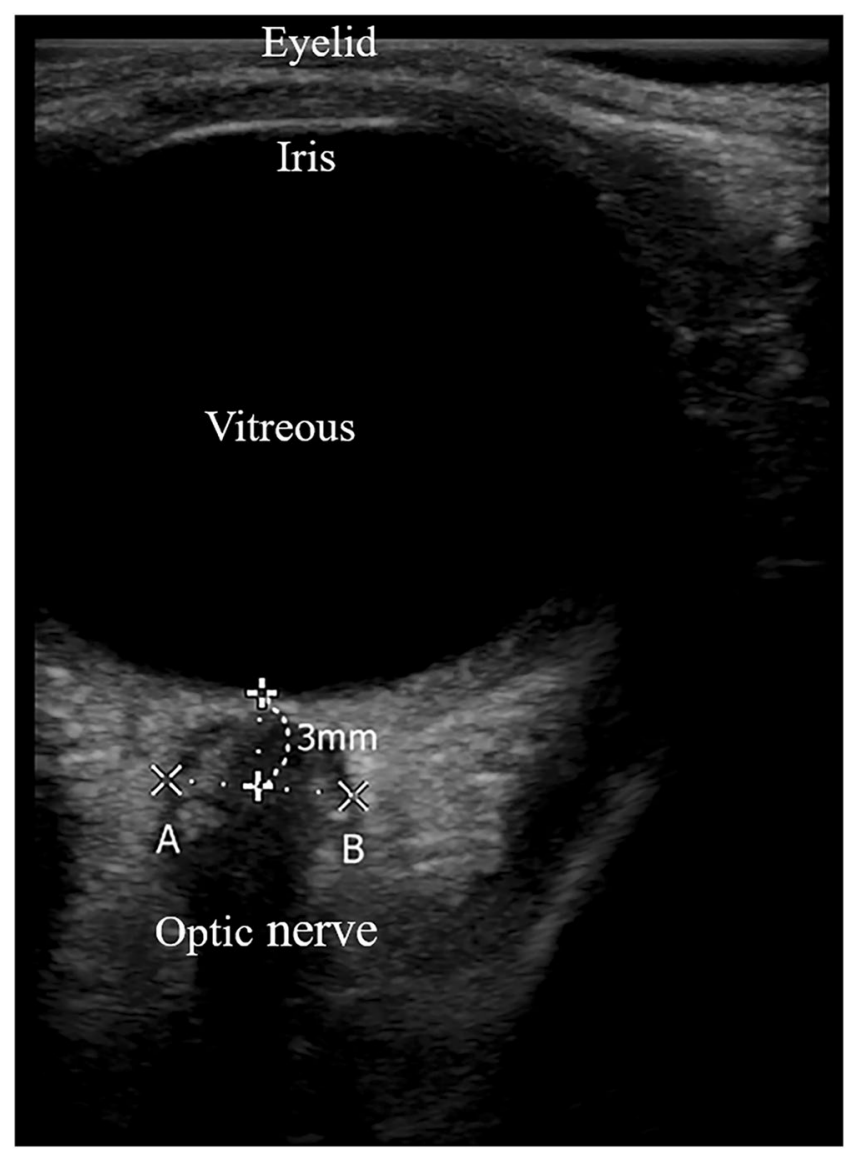

Figure 3. Measurement of optic nerve sheath diameter using ultrasonography. Axial plane images of the orbit were acquired through the optic nerve. Optic nerve sheath diameters were measured $3 \mathrm{~mm}$ posterior to the globe $(\mathbf{A}, \mathbf{B})$.

invasive arterial blood pressure, end-tidal carbon dioxide concentration, end-tidal sevoflurane concentrations, and peak inspiratory pressure were recorded at each time point.

Statistical analyses. Between-group differences in optic nerve sheath diameter were analysed. Based on previous findings, we considered a difference in optic nerve sheath diameter $>0.4 \mathrm{~mm}$ ( $10 \%$ of mean optic nerve sheath diameter in paediatric subjects under general anaesthesia $[4.3 \pm 0.3 \mathrm{~mm}])^{25}$ to be clinically relevant. Twelve subjects were required for each group at a significance level of $5 \%$ and power of $90 \%$. We enrolled 20 patients per group to compensate for withdrawals and observational variation.

The results for continuous variables are presented as mean (standard deviation) or median (interquartile range). The results for categorical variables are presented as numbers. Parametric data were analysed using independent $t$-tests. Nonparametric data were analysed using the Mann-Whitney U test. Fisher's exact tests or $\chi^{2}$ tests were used to evaluate categorical variables. Linear mixed models of the fixed and random effects between the two groups were used to examine the repeated optic nerve sheath diameter measurements. Intergroup comparisons of optic nerve sheath diameter changes over time included group-by-time interactions. Correlations between repeated measures were examined using an unstructured covariance matrix. The linear mixed models for optic nerve sheath diameter were adjusted for parameters that might affect optic nerve sheath diameter, intracranial pressure, or both (e.g., sex, age, height, weight, end-tidal carbon dioxide concentration, end-tidal sevoflurane concentration, and peak inspiratory pressure). Post-hoc analyses of optic nerve sheath diameter using the Bonferroni correction for multiple comparisons were also performed. Areas under the curve were calculated using the estimated mean (least squares mean) changes in optic nerve sheath diameter over time from the linear mixed models.

All statistical tests were two-tailed. P values $<0.05$ were considered to indicate statistically significant results. All analyses were performed using SPSS 23.0 (IBM Corp., Armonk, NY, USA) and SAS 9.4 (SAS Inc., Cary, NC, USA).

\section{Data availability}

The data that support the findings of this study are available from the corresponding author on reasonable request.

Received: 15 January 2019; Accepted: 11 March 2020;

Published online: 25 March 2020 


\section{References}

1. Taddio, A., Katz, J., Ilersich, A. L. \& Koren, G. Effect of neonatal circumcision on pain response during subsequent routine vaccination. Lancet (London, England) 349, 599-603, https://doi.org/10.1016/s0140-6736(96)10316-0 (1997).

2. Taddio, A., Goldbach, M., Ipp, M., Stevens, B. \& Koren, G. Effect of neonatal circumcision on pain responses during vaccination in boys. Lancet (London, England) 345, 291-292 (1995).

3. Schultz-Machata, A. M., Weiss, M. \& Becke, K. What's new in pediatric acute pain therapy? Current opinion in anaesthesiology 27, 316-322, https://doi.org/10.1097/aco.0000000000000074 (2014).

4. Willschke, H. et al. Epidural catheter placement in children: comparing a novel approach using ultrasound guidance and a standard loss-of-resistance technique. British journal of anaesthesia 97, 200-207, https://doi.org/10.1093/bja/ael121 (2006).

5. Kjeldgaard Pedersen, L., Nikolajsen, L., Rahbek, O., Uldall Duch, B. \& Moller-Madsen, B. Epidural analgesia is superior to local infiltration analgesia in children with cerebral palsy undergoing unilateral hip reconstruction. Acta orthopaedica 87, 176-182, https://doi.org/10.3109/17453674.2015.1113375(2016).

6. Moore, R. P., Wester, T., Sunder, R., Schrock, C. \& Park, T. S. Peri-operative pain management in children with cerebral palsy: comparative efficacy of epidural vs systemic analgesia protocols. Paediatric anaesthesia 23, 720-725, https://doi.org/10.1111/ pan.12187 (2013).

7. Brenn, B. R., Brislin, R. P. \& Rose, J. B. Epidural analgesia in children with cerebral palsy. Canadian journal of anaesthesia = Journal canadien d'anesthesie 45, 1156-1161, https://doi.org/10.1007/bf03012456 (1998).

8. Nolan, J., Chalkiadis, G. A., Low, J., Olesch, C. A. \& Brown, T. C. Anaesthesia and pain management in cerebral palsy. Anaesthesia 55, 32-41 (2000).

9. Lin, Y., Li, Q., Liu, J., Yang, R. \& Liu, J. Comparison of continuous epidural infusion and programmed intermittent epidural bolus in labor analgesia. Therapeutics and clinical risk management 12,1107-1112, https://doi.org/10.2147/tcrm.s106021 (2016).

10. Leone Roberti Maggiore, U. et al. Programmed intermittent epidural bolus versus continuous epidural infusion for pain relief during termination of pregnancy: a prospective, double-blind, randomized trial. International journal of obstetric anesthesia 25, 37-44, https://doi.org/10.1016/j.ijoa.2015.08.014 (2016).

11. Erdogan, M. A. et al. Patient-controlled Intermittent Epidural Bolus Versus Epidural Infusion for Posterior Spinal Fusion After Adolescent Idiopathic Scoliosis: Prospective, Randomized, Double-blinded Study. Spine 42, 882-886, https://doi.org/10.1097/ brs.0000000000001937 (2017).

12. Klumpner, T. T. et al. An in vitro evaluation of the pressure generated during programmed intermittent epidural bolus injection at varying infusion delivery speeds. Journal of clinical anesthesia 34, 632-637, https://doi.org/10.1016/j.jclinane.2016.06.017 (2016).

13. Lee, B. et al. Effect of caudal block using different volumes of local anaesthetic on optic nerve sheath diameter in children: a prospective, randomized trial. British journal of anaesthesia 118, 781-787, https://doi.org/10.1093/bja/aex078 (2017).

14. Padayachy, L. C., Padayachy, V., Galal, U., Gray, R. \& Fieggen, A. G. The relationship between transorbital ultrasound measurement of the optic nerve sheath diameter (ONSD) and invasively measured ICP in children: Part I: repeatability, observer variability and general analysis. Child's nervous system: ChNS: official journal of the International Society for Pediatric Neurosurgery 32, 1769-1778, https://doi.org/10.1007/s00381-016-3067-5 (2016).

15. Choi, S. H. et al. Ultrasonography of the optic nerve sheath to assess intracranial pressure changes after ventriculo-peritoneal shunt surgery in children with hydrocephalus: a prospective observational study. Anaesthesia 70, 1268-1273, https://doi.org/10.1111/ anae.13180 (2015).

16. Le, A. et al. Bedside sonographic measurement of optic nerve sheath diameter as a predictor of increased intracranial pressure in children. Annals of emergency medicine 53, 785-791, https://doi.org/10.1016/j.annemergmed.2008.11.025 (2009).

17. Groenewald, C. B., Rabbitts, J. A., Schroeder, D. R. \& Harrison, T. E. Prevalence of moderate-severe pain in hospitalized children. Paediatric anaesthesia 22, 661-668, https://doi.org/10.1111/j.1460-9592.2012.03807.x (2012).

18. Hogan, Q. Distribution of solution in the epidural space: examination by cryomicrotome section. Regional anesthesia and pain medicine 27, 150-156 (2002).

19. Kaynar, A. M. \& Shankar, K. B. Epidural infusion: continuous or bolus? Anesthesia and analgesia 89, 534 (1999).

20. Carvalho, B., George, R. B., Cobb, B., McKenzie, C. \& Riley, E. T. Implementation of Programmed Intermittent Epidural Bolus for the Maintenance of Labor Analgesia. Anesthesia and analgesia 123, 965-971, https://doi.org/10.1213/ane.0000000000001407 (2016).

21. Ferrer, L. E., Romero, D. J., Vasquez, O. I., Matute, E. C. \& Van de Velde, M. Effect of programmed intermittent epidural boluses and continuous epidural infusion on labor analgesia and obstetric outcomes: a randomized controlled trial. Archives of gynecology and obstetrics 296, 915-922, https://doi.org/10.1007/s00404-017-4510-x (2017).

22. Lim, Y., Sia, A. T. \& Ocampo, C. Automated regular boluses for epidural analgesia: a comparison with continuous infusion. International journal of obstetric anesthesia 14, 305-309, https://doi.org/10.1016/j.ijoa.2005.05.004 (2005).

23. Helmke, K. \& Hansen, H. C. Fundamentals of transorbital sonographic evaluation of optic nerve sheath expansion under intracranial hypertension. I. Experimental study. Pediatric radiology 26, 701-705 (1996).

24. Hansen, H. C. \& Helmke, K. Validation of the optic nerve sheath response to changing cerebrospinal fluid pressure: ultrasound findings during intrathecal infusion tests. Journal of neurosurgery 87, 34-40, https://doi.org/10.3171/jns.1997.87.1.0034 (1997).

25. Min, J. Y. et al. Ultrasonographic assessment of optic nerve sheath diameter during pediatric laparoscopy. Ultrasound in medicine \& biology 41, 1241-1246, https://doi.org/10.1016/j.ultrasmedbio.2015.01.009 (2015).

26. Dubost, C. et al. Optic nerve sheath diameter used as ultrasonographic assessment of the incidence of raised intracranial pressure in preeclampsia: a pilot study. Anesthesiology 116, 1066-1071, https://doi.org/10.1097/ALN.0b013e318246ea1a (2012).

27. Moretti, R. \& Pizzi, B. Ultrasonography of the optic nerve in neurocritically ill patients. Acta anaesthesiologica Scandinavica 55, 644-652, https://doi.org/10.1111/j.1399-6576.2011.02432.x (2011).

28. Dubourg, J., Javouhey, E., Geeraerts, T., Messerer, M. \& Kassai, B. Ultrasonography of optic nerve sheath diameter for detection of raised intracranial pressure: a systematic review and meta-analysis. Intensive care medicine 37, 1059-1068, https://doi.org/10.1007/ s00134-011-2224-2 (2011).

29. Geeraerts, T., Merceron, S., Benhamou, D., Vigue, B. \& Duranteau, J. Non-invasive assessment of intracranial pressure using ocular sonography in neurocritical care patients. Intensive care medicine 34, 2062-2067, https://doi.org/10.1007/s00134-008-1149-x (2008).

30. Girisgin, A. S. et al. The role of optic nerve ultrasonography in the diagnosis of elevated intracranial pressure. Emergency medicine journal: EMJ 24, 251-254, https://doi.org/10.1136/emj.2006.040931 (2007).

31. Padayachy, L. C., Padayachy, V., Galal, U., Pollock, T. \& Fieggen, A. G. The relationship between transorbital ultrasound measurement of the optic nerve sheath diameter (ONSD) and invasively measured ICP in children.: Part II: age-related ONSD cutoff values and patency of the anterior fontanelle. Child's nervous system: ChNS: official journal of the International Society for Pediatric Neurosurgery 32, 1779-1785, https://doi.org/10.1007/s00381-016-3068-4 (2016).

32. Steinborn, M. et al. Normal values for transbulbar sonography and magnetic resonance imaging of the optic nerve sheath diameter (ONSD) in children and adolescents. Ultraschall in der Medizin (Stuttgart, Germany: 1980) 36, 54-58, https://doi. org/10.1055/s-0034-1385012 (2015). 


\section{Author contributions}

B.L.: Patient recruitment, data collection, and analysis, and preparation of the first draft of the manuscript. J.H.L.: Data collection and manuscript revision. M.-S.K.: Data collection. S.J.K.: Data collection. J.S.: Data collection. D.H.K.: Data collection. Y.S.C.: Study design, randomisation, and preparation of the final version of the manuscript. All authors reviewed the manuscript.

\section{Competing interests}

The authors declare no competing interests.

\section{Additional information}

Correspondence and requests for materials should be addressed to Y.S.C.

Reprints and permissions information is available at www.nature.com/reprints.

Publisher's note Springer Nature remains neutral with regard to jurisdictional claims in published maps and institutional affiliations.

(c) (i) Open Access This article is licensed under a Creative Commons Attribution 4.0 International License, which permits use, sharing, adaptation, distribution and reproduction in any medium or format, as long as you give appropriate credit to the original author(s) and the source, provide a link to the Creative Commons license, and indicate if changes were made. The images or other third party material in this article are included in the article's Creative Commons license, unless indicated otherwise in a credit line to the material. If material is not included in the article's Creative Commons license and your intended use is not permitted by statutory regulation or exceeds the permitted use, you will need to obtain permission directly from the copyright holder. To view a copy of this license, visit http://creativecommons.org/licenses/by/4.0/.

(C) The Author(s) 2020 